\title{
HD 75289Ab revisited
}

\section{Searching for starlight reflected from a hot Jupiter}

\author{
F. Rodler ${ }^{1,2}$, M. Kürster ${ }^{1}$, and T. Henning ${ }^{1}$ \\ 1 Max-Planck-Institut für Astronomie, Königstuhl 17, 69117 Heidelberg, Germany \\ e-mail: rodler@mpia.de \\ 2 Institut für Astronomie, Universität Wien, Türkenschanzstrasse 17, 1180 Vienna, Austria
}

Received 30 November 2007 / Accepted 29 February 2008

\section{ABSTRACT}

\begin{abstract}
Aims. We attempt to detect starlight reflected from a hot Jupiter, orbiting the main-sequence star HD 75289Ab. We report a revised analysis of observations of this planetary system presented previously by another research group.

Methods. We analyze high-precision, high-resolution spectra, collected over four nights using UVES at the VLT/UT2, by way of data synthesis. We try to interpret our data using different atmospheric models for hot Jupiters.

Results. We do not find any evidence for reflected light, and, therefore, establish revised upper limits to the planet-to-star flux ratio at the $99.9 \%$ significance level. At high orbital inclinations, where the best sensitivity is attained, we can limit the relative reflected radiation to be less than $\epsilon=6.7 \times 10^{-5}$ assuming a grey albedo, and $\epsilon=8.3 \times 10^{-5}$ assuming an Class IV function, respectively. This implies a geometric albedo smaller than $p=0.46$ and $p=0.57$, for the grey albedo and the Class IV albedo shape, respectively, assuming a planetary radius of $1.2 R_{\mathrm{Jup}}$.
\end{abstract}

Key words. methods: data analysis - techniques: radial velocities - stars: individual: HD 75289A - stars: planetary systems

\section{Introduction}

Since the detection of the first exoplanet orbiting a solar-type star, more than 270 exoplanets have been detected. So-called hot Jupiters - giant planets only a few solar radii away from their host stars - provide the opportunity to detect starlight reflected from these planets. Five extended campaigns for the search for reflected light were completed by different groups, all resulting in non-detections (e.g. Leigh et al. 2003b, and references therein). Upper limits to the planet-to-star flux ratio and to the geometric albedo of these planets were established, which provided important constraints to models of the planetary atmospheres by Sudarsky et al. (2000, 2003). As a result, models that predicted a high reflectivity for the planetary atmosphere could be ruled out for some of the studied planets.

Here, we present a reanalysis of observations of the planetary system of HD 75289A, conducted by Leigh et al. (2003a), over four nights in January 2003, using the UVVisual Echelle Spectrograph (UVES) mounted on the VLT/UT2 at Cerro Paranal in Chile. These authors attempted to detect starlight reflected from the hot Jupiter, but were unable to find evidence for the planetary signal: they placed a $99.9 \%$ confidence upper limit on the planet-to-star flux ratio of $4.17 \times 10^{-5}$, for the spectral range $\lambda=402.33$ to $522.13 \mathrm{~nm}$, and for an orbital inclination $i=60^{\circ}$.

We noticed, however, that this upper limit was based on erroneous orbital phase information for the planet. In this article, we correct the upper limit to the planet-to-star flux ratio determined by Leigh et al. (2003), using using our implementation of the modeling approach introduced by Charbonneau et al. (1999). Section 2 describes the basic ideas of the search for reflected light. Section 3 provides a brief overview of the science data, while Sect. 4 provides a detailed description of sophisticated data processing implemented by using the data modeling approach. Finally, in Sect. 5 we present our corrected upper limits to the planet-to-star flux ratio.

\section{Reflected light}

\subsection{Photometric variations}

For exoplanets, the enormous brightness contrast between the star and the planet constitutes considerable challenge when attempting to observe some kind of direct signal from the planet. For close-in planets such as hot Jupiters, the main contribution to the optical flux originates in the reflected starlight and not the intrinsic luminosity (Seager et al. 2000). High-resolution spectroscopy in the optical utilizes the fact that the observed spectrum reflected from the planet is essentially a copy of the rich stellar absorption-line spectrum. Basically, this spectrum is shifted in wavelength according to the orbital radial velocity (RV) of the planet and scaled down in brightness by a factor of a few times $10^{4}$ for hot Jupiters. According to Charbonneau et al. (1999), the amount of starlight reflected from a planet which is fully illuminated can be described by

$\epsilon(\lambda)=p(\lambda)\left(\frac{R_{\mathrm{p}}}{a}\right)^{2}$,

where $p(\lambda)$ denotes the geometric albedo of the planet as a function of the wavelength $\lambda, R_{\mathrm{p}}$ the planetary radius and $a$ the star-planet separation. Figure 1 shows different albedo spectra, derived using different planetary-atmosphere models from Sudarsky et al. (2000). The planetary radius of HD 75289Ab is unknown; it can, however, be estimated from the transiting planets, which provide an exact determination of their masses and 


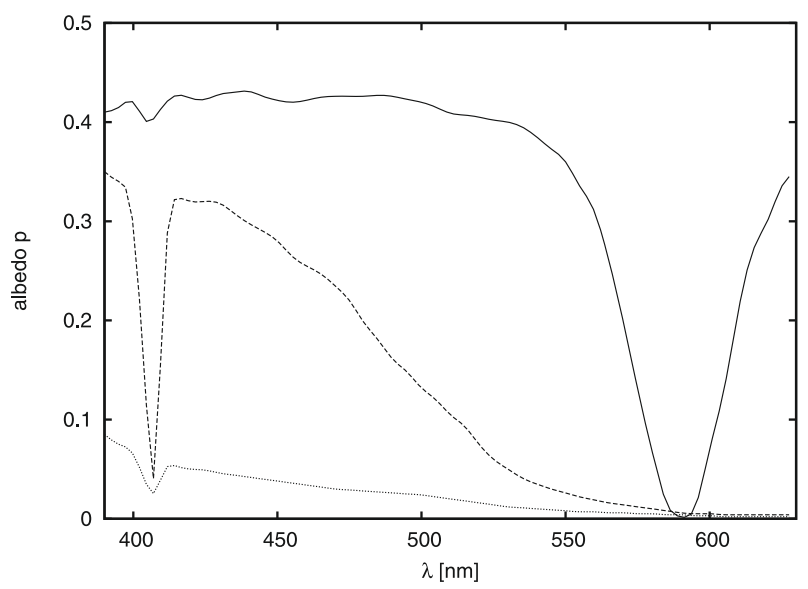

Fig. 1. Different albedo spectra of atmospheric models (taken from Sudarsky et al. 2000) are shown. The irradiated (dots) and isolated (dashed) Class IV models describe atmospheres of planets with temperatures $T_{\mathrm{eff}} \approx 1300 \mathrm{~K}$. In contrast to the isolated model, the irradiated model assumes that no reflective clouds exist in the upper layer of the planetary atmosphere, which results in a very low geometric albedo. The solid line depicts a Class V roaster, describing the atmosphere of a planet with $T_{\text {eff }} \geq 1500 \mathrm{~K}$, having a highly reflective silicate cloud deck in the upper layers of the atmosphere.

radii (see Sect. 2.3). The orbital radius can be tightly constrained using Kepler's third law.

In most cases, the planet does not appear to be fully illuminated. Consequently, the observed reflected light is reduced, depending on the model describing the scattering behavior of the atmosphere, its orbital inclination $i \in\left[0^{\circ}, 90^{\circ}\right]$ and the orbital phase $\phi \in[0,1]$ of the planet. We note that we adopt the convention that $\phi=0$ represents inferior conjunction of the planet (for $i=90^{\circ}$, it would be the transit position). We apply an empirical scattering model of the atmospheres of Jupiter and Venus (Hilton 1992), which can be approximated by

$\mu(\phi, i)=10^{-0.4 \zeta(\alpha)}$,

where

$\zeta(\alpha)=0.09\left(\frac{1.8 \alpha}{\pi}\right)+2.39\left(\frac{1.8 \alpha}{\pi}\right)^{2}-0.65\left(\frac{1.8 \alpha}{\pi}\right)^{3}$

and the phase angle $\alpha$ :

$\cos \alpha=-\sin i \cos 2 \pi \phi$.

Finally, the flux of the reflected light from the planet at the orbital phase $\phi$ follows from Eqs. (1) and (2):

$f(\phi, i, \lambda)=\epsilon(\lambda) \mu(\phi, i)$.

\subsection{Doppler shifts}

The planet orbiting its host star produces not only a flux variation (Eq. (5)), but also a Doppler shift of the stellar spectrum reflected from the planet. The RV semi-amplitude $K_{\mathrm{p}}$ of that shift depends on the orbital inclination $i$, which is unknown for most of the non-transiting planets. $K_{\mathrm{p}}$ can be expressed by

$K_{\mathrm{p}}=K_{\mathrm{s}} \frac{M_{\mathrm{s}}}{M_{\mathrm{p}} \sin i} \sin i$,

where $K_{\mathrm{s}}$ is the RV semi-amplitude of the star, and $M_{\mathrm{S}}$ and $M_{\mathrm{p}} \sin i$ are the stellar mass and the minimum mass of the planet,
Table 1. Parameters of the star HD 75289A and its planetary system.

\begin{tabular}{lrll}
\hline \hline Parameter & Value & Error & Ref. \\
\hline Star: & & & \\
Spectral type & $\mathrm{G} 0 \mathrm{~V}$ & & G89 \\
$V(\mathrm{mag})$ & 6.35 & & VF05 \\
$d(\mathrm{pc})$ & 28.94 & 0.47 & VF05 \\
$M_{\mathrm{s}}\left(M_{\odot}\right)$ & 1.23 & 0.10 & VF05 \\
$R_{\mathrm{s}}\left(R_{\odot}\right)$ & 1.249 & 0.022 & VF05 \\
$P_{\text {rot }}(\mathrm{d})$ & 16.0 & 3.0 & $\mathrm{U} 00$ \\
$v \sin i\left(\mathrm{~km} \mathrm{~s}^{-1}\right)$ & 4.14 & & B06 \\
Age $(\mathrm{Gyr})$ & 2.2 & & VF05 \\
\hline Planet: & & & \\
$M_{\mathrm{p}} \sin i\left(M_{\text {Jup }}\right)$ & 0.467 & 0.041 & $\mathrm{~B} 06$ \\
$a(\mathrm{AU})$ & 0.0482 & 0.0028 & $\mathrm{~B} 06$ \\
$e$ & 0.034 & 0.029 & $\mathrm{~B} 06$ \\
$K_{\mathrm{s}}\left(\mathrm{km} \mathrm{s} \mathrm{s}^{-1}\right)$ & 0.0549 & 0.0019 & $\mathrm{~B} 06$ \\
Orbital period (d) & 3.509267 & 0.000064 & $\mathrm{~B} 06$ \\
$T_{\phi=0}(\mathrm{JD})$ & 2450829.872 & 0.038 & B06 \\
\hline
\end{tabular}

References: B06 = Butler et al. (2006, and references therein), G89 = Gratton et al. (1989), U00 = Udry et al. (2000), VF05 = Valenti \& Fischer (2005).

respectively. The largest possible amplitude $K_{\mathrm{p}, \max }$ occurs at the orbital inclination $i=90^{\circ}$.

The instantaneous RV shift of the planetary signal with respect to the star depends on the orbital phase $\phi$,

$V_{\mathrm{p}}=K_{\mathrm{p}} \sin 2 \pi \phi$.

\section{3. $H D 75289 A$ and its planet}

High-precision RV measurements revealed the existence of a hot Jupiter orbiting the G0-type main-sequence star HD 75289A (Udry et al. 2000). Table 1 summarizes the parameters of the planet and its host star. We note in passing that the system also contains a faint low-mass stellar component, separated by $\approx 621$ AU from the primary (Mugrauer et al. 2004).

The planetary system of HD 75289A seemed to bear the opportunity to detect the reflected starlight for the following reasons: first, the brightness of HD 75289A in the visual $(V=$ $6.35 \mathrm{mag}$ ) enables a large amount of high-S/N spectra to be acquired within a short period of time, which helps to reduce photon noise, the dominant noise source. Second, the amount of starlight received from this planet is likely to be high due to the extremely small distance to its host star of only $a=0.048 \mathrm{AU}$ (cf. Eq. (1)). Third, a G0 V star shows a rich absorption-line spectrum at optical wavelengths, a prerequisite for the datasynthesis approach outlined in Sect. 4.

Using data of the stellar mass $M_{\mathrm{s}}$, the planetary minimum mass $M_{\mathrm{p}} \sin i$, and the RV semi-amplitude of the reflex motion of the star $K_{\mathrm{s}}$, the maximum possible RV semi-amplitude of the planet can be determined (from Eq. (6)) to be $K_{\mathrm{p}, \max }=$ $148.9 \pm 15.8 \mathrm{~km} \mathrm{~s}^{-1}$. An estimate of the planetary radius $R_{\mathrm{p}}$ can be obtained from a comparison of the radii determined for other hot Jupiters with the transit method. To this end, we selected a subsample of known transiting hot Jupiters (Burrows et al. 2007, and references therein), based on the following criteria: (i) planet mass between the minimum mass of HD 75289Ab and 2.3 times this value (for random orientation of the planetary orbit there is a $90 \%$ confidence that the true mass of HD 75289Ab lies in this interval); (ii) orbital radii similar to that of HD 75289Ab within 20\%; (iii) host star spectral types close to G0 V (F8 V-G1 V). The resulting group of objects contains OGLE 10b, OGLE 111b, 
Table 2. Journal of observations. The UTC times and the orbital phases of the planets are shown. Note that $\phi_{\text {Leigh }}$ indicates the erroneous phases applied by Leigh et al. (2003), while $\phi_{\text {corr }}$ refers to the corrected ones. The number of spectra obtained is given in the last column. Ephemerides used are: orbital period $=3.509267 \mathrm{~d}, T_{\phi=0}(\mathrm{BJD})=2450829.872$ (Butler et al. 2006, and references therein).

\begin{tabular}{|c|c|c|c|c|c|c|c|}
\hline Night & UTC start & $\phi_{\text {Leigh }}$ & $\overline{\phi_{\text {corr }}}$ & UTC end & $\overline{\phi_{\text {Leigh }}}$ & $\phi_{\text {corr }}$ & $\overline{N_{\text {spectra }}}$ \\
\hline 1 & $2003 / 01 / 14 \quad 01: 00$ & 0.39 & 0.68 & $2003 / 01 / 14 \quad 09: 30$ & 0.50 & 0.79 & 188 \\
\hline 2 & $2003 / 01 / 15 \quad 00: 57$ & 0.68 & 0.97 & 2003/01/15 09:22 & 0.78 & 0.07 & 173 \\
\hline 3 & $2003 / 01 / 21 \quad 02: 40$ & 0.41 & 0.70 & $2003 / 01 / 21 \quad 09: 35$ & 0.49 & 0.78 & 183 \\
\hline 4 & $2003 / 01 / 22 \quad 03: 36$ & 0.71 & 0.00 & 2003/01/22 09:41 & 0.78 & 0.07 & 140 \\
\hline
\end{tabular}

HAT-P-1b, XO-1b, HD 209458b. From this sample, we estimated the planetary radius of HD 75289Ab as the average of the radii of these five hot Jupiters finding $R_{\mathrm{p}}=1.2 \pm 0.14 R_{\mathrm{Jup}}$. The uncertainty corresponds to the scatter in the above sample of transit radii.

\section{Data}

We retrieved 684 high-resolution and high-S/N spectra files from the ESO science archive, along with the calibration files. The observations were conducted over four nights by Leigh et al. (2003a) with UVES mounted on the VLT/UT2. The blue arm of the spectrograph was used with the single EEV CCD-44 array detector centered at wavelength $\lambda=475.8 \mathrm{~nm}$ providing 25 full orders and covering the wavelength range $\lambda=402.3$ to $522.1 \mathrm{~nm}$. The integration times were adapted to the seeing such that a count rate not exceeding 40000 ADU per pixel was achieved, insuring high $\mathrm{S} / \mathrm{N}$ levels and at the same time staying clear of the saturation level. The effective resolving power was $R=43000$. More details of the observations can be found in Leigh et al. (2003a).

These authors selected the observing dates for the best visibility of the planet, which is in the orbital phase range $\phi=0.30$ to 0.45 and 0.55 to 0.70 (close to superior conjunction), but excluding phases about 0.50 , where the absorption-line systems of the star and the planet blend. However, we found that a oneday error in the date used for the phase calculation must have been made. This resulted in the fact that the planet was not observed at the optimum phases. In particular, during two nights the observations were taken close to the inferior conjunction of the planet, where it is hardly visible. Furthermore, the same erroneous phases were used in the subsequent data analysis. Consequently, one can expect the corrected upper limit to the planet-to-star flux ratio to be smaller than the value determined by Leigh et al. Table 2 shows the journal of observations with the corresponding phases of the planet (erroneous and corrected ones).

\subsection{Data reduction}

The data were reduced using a UVES data-reduction pipeline developed by ourselves. For high-quality flat-field correction, 228 and 369 flat-field exposures were combined for the first two nights and for the last two ones, respectively. Furthermore, 90 bias exposures were combined. We retrieved 25 orders of 3000 pixels from each echelle spectrum. No order merging was applied. Furthermore, we identified cosmic-ray hits by way of the following procedure: for each spectrum, we compared the flux in every pixel with the median flux of the same pixel in the three predecessor and the three successor spectra, which had been scaled to the same flux as the spectrum under consideration. We flagged those pixels where the difference exceeded $6 \sigma$ as cosmic-ray hits. These pixels were then excluded from further analysis.

We discarded the most weakly exposed regions of each echelle order (the first 300 pixels as well as the last 100 pixels). To speed up the data analysis, we co-added spectra into sufficiently narrow phase bins such that phase smearing of the stellar absorption lines originating from stellar RV variation, sub-pixel shifts (see Sect. 4.1), and the barycentric motion of the Earth, remained below $0.04 \mathrm{~km} \mathrm{~s}^{-1}$. An additional criterion for the size of the phase bins was that the unseen planetary lines did not suffer from smearing in excess of $2 \mathrm{~km} \mathrm{~s}^{-1}$. In these ways, we reduced the number of spectra from 684 to 210 .

\section{Data analysis: the data synthesis method}

We model the starlight reflected from the planet as a copy of the stellar spectrum, strongly scaled down in brightness and Doppler-shifted according to the orbital motion of the planet. The HD 75289A spectra have an average S/N of 300 to 600 per dispersion element. With the expectation the planet-to-star flux ratios of the order of a few times $10^{-5}$, it is clear that the reflected spectrum from the planet is deeply buried in the noise of the stellar spectrum. The weak planetary signal is increased by the large number of spectra, and more importantly, by the combination of the approximately 1500 absorption lines, achieved using the data-synthesis method described below.

\subsection{Step 1: construction of the superspectrum}

To create a high $\mathrm{S} / \mathrm{N}$, virtually planet-free superspectrum, we first create copies of the original, unmodified object spectra. These copies are then co-aligned with respect to the barycentric velocity of the Earth and the radial velocity of the star, and finally summed up. Here, it is important that the observing dates cover different orbital phases well distributed such that the planetary signal in the superspectrum is washed out. In the case of the HD 75289A data, nearly half of the observations were conducted in the orbital phase range $\phi=0.97$ to 0.07 (Table 2), where the planet was close to inferior conjunction and therefore at its faintest (e.g. for an orbital inclination of $i=60^{\circ}$, the planet appears about 12 times fainter at the inferior conjunction, relative to its maximum brightness). Consequently, we used only these "planet-free" spectra to create the superspectrum.

We found that imperfections in the dispersion solution (subpixel shifts and stretches/contractions) of the different spectra led to broadening of absorption lines in the superspectrum. We assume that these shifts originate mostly from errors of the wavelength calibration and probably to some extent from guiding errors and variations in the instrumental profile. Consequently, we correct each spectrum for these wavelength errors before producing a new version of the superspectrum. To this end, we first divide each order of the modified copies of the object spectra into chunks of size $N$ pixel, and determine the central wavelength $\lambda_{i}$ 
of each chunk $i$. For the data analysis of HD 75289A, we set $N=120$, which creates 22 chunks per order. By means of the Brent algorithm (Press et al. 1992), we determine for each chunk the sub-pixel shift $s_{i}$ between the superspectrum and the modified copy of the object spectrum under consideration. Then, we shift each pixel of the modified copy by a value determined by calculating a spline through all pairs of $\lambda_{i}, s_{i}$ per spectral order. Finally, we add up the re-adjusted co-aligned versions of the copies of the object spectra and get an improved version of the superspectrum.

\subsection{Step 2a: modeling the stellar signal ...}

Each of the original, unmodified object spectra is modeled using the improved superspectrum. In this initial step, we ignore the presence of the faint planetary signal in the data. First the superspectrum (model) is corrected for a general linear trend in flux. Second, the model is shifted according to the barycentric velocity of the Earth, the radial velocity of the star, and the aforementioned shifts and stretches/contractions in the sub-pixel regime, so that the positions of absorption lines of the object spectrum and the model are matched. This is achieved using a chunkBrent-spline-approach similar to that described in the previous paragraph. We note that in all analysis steps, modifications are exclusively applied to the model, but the object spectrum are used in their original version, i.e. the data are unchanged.

At the third stage, the model is scaled chunkwise with respect to the object spectrum. As we now compare the scaled model with each object spectrum, we notice that the widths and depths of the absorption lines differ slightly. These differences originate most likely in the aforementioned effects of residuals wavelength calibration errors, guiding errors, and variations in the instrumental profile, and can be corrected by adding a scaled version of the second derivative of the object spectrum to the model. The scaling factor is determined via $\chi^{2}$ minimization (fourth stage). After this, the model is renormalized. The final stage is to iterate twice over all these four processes to improve the model describing the stellar spectrum.

\subsection{Step $2 b:$... and the planetary signal}

For the model of the planetary signal, we use a copy of the improved model of the stellar spectrum, but scaled down by the factors $\epsilon(\lambda) \mu(\phi, i)$ and shifted by velocity $V_{\mathrm{p}}\left(K_{\mathrm{p}}, \phi\right)$ with respect to the stellar spectrum. Hence, the two free parameters are the planet-to-star flux ratio for the fully-illuminated planet $\epsilon(\lambda)$, and the orbital inclination $i$, which corresponds to the RV semiamplitude of the planet $K_{\mathrm{p}}=K_{\mathrm{p}, \max } \sin i=148.9 \sin i \mathrm{~km} \mathrm{~s}^{-1}$.

\subsection{Step 3: evaluation of the models}

We are now ready to add this planetary signal to the improved model $T$ of the stellar spectrum and consequently construct the model $M$ describing the spectrum of the star plus the reflected one from the planet. For each pixel $k, M$ is given by

$$
M_{k}=\frac{T_{k}\left(\lambda_{k}\right)+\epsilon\left(\lambda_{k}\right) \mu(\phi, i) T_{k}\left\{\lambda_{k}\left[1+V_{p}\left(K_{\mathrm{p}}, \phi\right) c^{-1}\right]\right\}}{1+\epsilon\left(\lambda_{k}\right) \mu(\phi, i)},
$$

where $c$ denotes the speed of light.

Varying $K_{\mathrm{p}}$ and $\epsilon(\lambda)$, we finally search for the best-fit model $M$ to all the object spectra by $\chi^{2}$ minimization. The search range for the RV semi-amplitude comprised $K_{\mathrm{p}}=40$ to $180 \mathrm{~km} \mathrm{~s}^{-1}$ (corresponding to orbital inclinations $i=15^{\circ}$ to $90^{\circ}$, plus twice the error of $K_{\mathrm{p} \text {,max }}$; see Table 1) with a step width of $3 \mathrm{~km} \mathrm{~s}^{-1}$. This was a good compromise between computing time and sampling the average absorption line profile with the FWHM of $\approx 15 \mathrm{~km} \mathrm{~s}^{-1}$. Using simulations, we found that for small inclinations of the planetary orbit, where the planets appear only slightly illuminated, the method is unable to detect Jupiter-size objects with very high geometric albedos.

\subsection{Step 4: determination of the confidence level}

Once the best model $M\left[K_{\mathrm{p}}, \epsilon(\lambda)\right]$ has been evaluated, we determine the confidence level of the $\chi^{2}$ minimum by applying the bootstrap randomisation method (e.g. Kürster et al. 1997). Retaining the orbital phases, we randomly redistribute the observed spectra amongst the phases, thereby creating $N$ different data sets. Any signal present in the original data is now scrambled in these artificial data sets. For all these randomised data sets, we again evaluate the model for the two free parameters, and locate the best fit with its specific $\chi^{2}$ minimum. We set $m$ to be the number of best-fit models to the $N$ randomised data sets that have a minimum $\chi^{2}$ less or equal than the minimum $\chi^{2}$ found for the original data set. The confidence level can then be estimated by $\approx 1-m / N$.

\section{Results and discussion}

Applying the data synthesis method to the HD 75289A data, we adopted the following approximations to the atmospheric models by Sudarsky et al. (2000).

(i) We adopted a grey-albedo model to resemble the Class V model, which describes the atmospheres of hot Jupiters with temperatures $>1500 \mathrm{~K}$. As can be seen in Fig. 1, this was a valid approximation for our observed wavelength range (402 to $522 \mathrm{~nm}$ ).

(ii) We also considered the two Class IV models for hot Jupiters with temperatures around $1300 \mathrm{~K}$. The irradiated model predicts a very low reflectivity due to the lack of silicates in the upper layers of the planetary atmosphere, while the isolated model predicts high reflectivity due to the existence of silicate clouds in the upper decks of the planetary atmosphere. We found that both Class IV models show a similar trend for the albedo function. Therefore we considered only that trend in further modeling.

\subsection{Grey albedo model (corresponding to Class V)}

Our data analysis, using a grey albedo, revealed a $\chi^{2}$-minimum at a planet-to-star flux ratio $\epsilon(\lambda)=2.9 \times 10^{-5}$, and an RV semiamplitude $K_{\mathrm{p}}=121 \mathrm{~km} \mathrm{~s}^{-1}$ corresponding to an orbital inclination of $i=54^{\circ}$. Figure 2 (upper panel) shows a $\chi^{2}$ contour map. However, using bootstrap randomisation with 3000 trial data sets we found that this $\chi^{2}$-minimum was uncertain with a false-alarm probability (FAP) of 19\%; we do not therefore consider this as a detection of reflected light from the planet.

\subsection{Class IV model}

The analysis using the Class IV albedo model did not yield any evidence for reflected light from the hot Jupiter HD 75289Ab. We found that the $\chi^{2}$-minimum at a planet-to-star flux ratio $\epsilon(\lambda)=3.3 \times 10^{-5}$ and an RV semi-amplitude $K_{\mathrm{p}}=119 \mathrm{~km} \mathrm{~s}^{-1}$, which corresponds to an orbital inclination $i=53^{\circ}$. Figure 2 

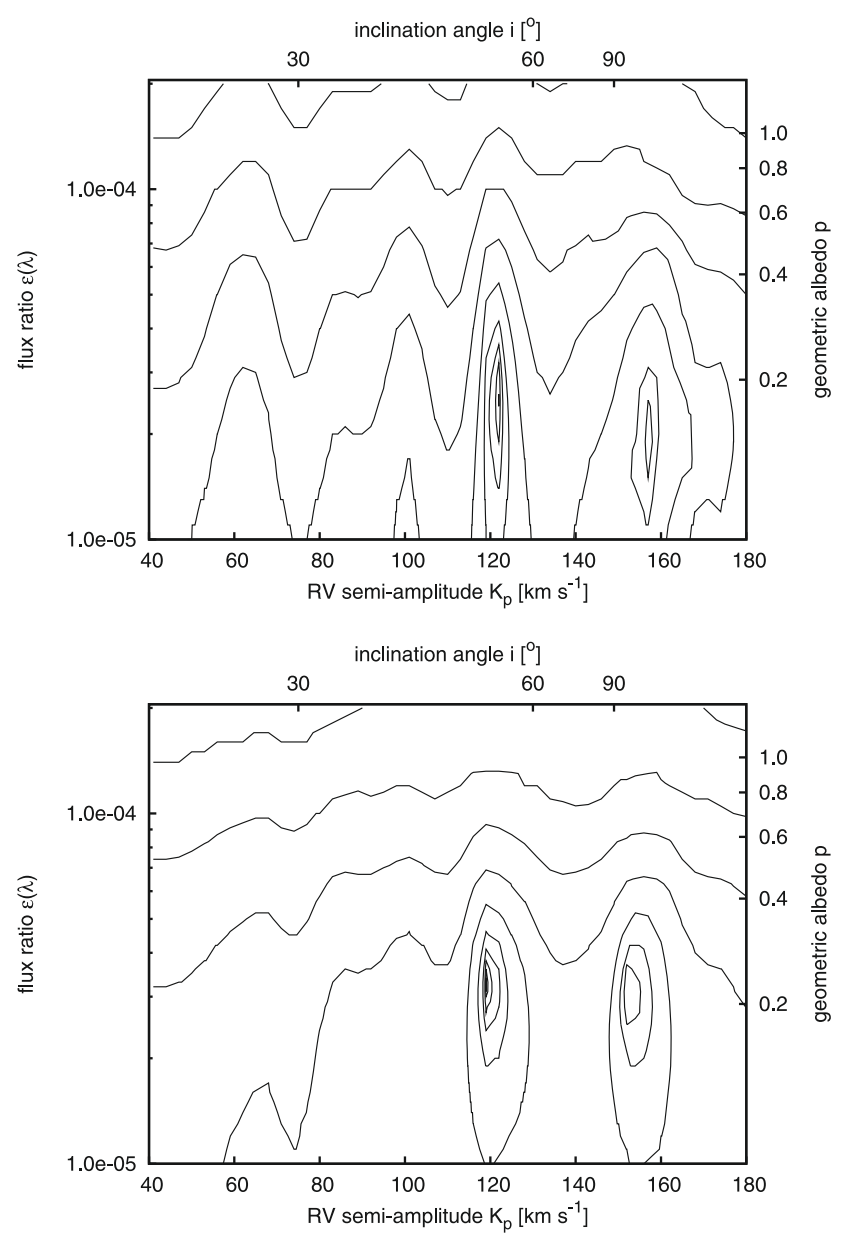

Fig. 2. Contour maps of $\chi^{2}$ normalised to $\chi_{\min }^{2}$ for the model parameters $K_{\mathrm{p}}$ and $\epsilon(\lambda)$ showing the result of the analysis of the HD 75289A data for two different atmospheric models. For better visualisation the $\chi^{2}$ contour levels follow the sequence $\chi_{k}^{2}=\chi_{\text {min }}^{2}+0.1 \chi_{\text {min }}^{-2} \mathrm{e}^{k-1}$ for $k>0$. Upper panel: for the planetary model, we assumed a Venuslike phase function and a grey albedo. The minimum $\chi^{2}$ was found at $K_{\mathrm{p}}=121 \mathrm{~km} \mathrm{~s}^{-1}$ and $\epsilon(\lambda)=2.9 \times 10^{-5}$. An analysis with 3000 trials revealed that this feature is uncertain with a false alarm probability of 19\%. Lower panel: for the planetary model, we assumed a Venuslike phase function and a class IV albedo function. The minimum $\chi^{2}$ was found at $K_{\mathrm{p}}=119 \mathrm{~km} \mathrm{~s}^{-1}$ and $\epsilon(\lambda)=3.3 \times 10^{-5}$. An analysis with 3000 trials revealed that this feature is uncertain with a false alarm probability of $24 \%$. We note that the corresponding geometric albedo $p$ is shown under the assumption that $R_{\mathrm{p}}=1.2 R_{\text {Jup }}$ (the right-hand $y$-axis).

(lower panel) shows a contour map of that feature. From bootstrap randomisation with 3000 trials we found that this feature was uncertain with a FAP of $24 \%$.

\subsection{Upper limits}

Obviously, the amount of data for when the planet was observed during its bright phases was insufficient to measure the planetary signal. The question is now: at what planet-to-star flux ratio $\epsilon(\lambda)$ would we be able to detect significantly the planetary signal using the present data? To this end, we constructed data sets by adding an artificial planetary signal to the original data. To produce the planetary signal, we explored the range of $K_{\mathrm{p}} \in[40,180] \mathrm{km} \mathrm{s}^{-1}$ and $\epsilon(\lambda) \in\left[2 \times 10^{-5}, 2.5 \times 10^{-4}\right]$. For each data set, the simulated planetary signal was a copy of the object spectrum, but shifted by $V_{\mathrm{p}}\left(K_{\mathrm{p}}, \phi\right)$ and scaled down by the factor $f(\phi, i, \lambda)$, which includes the albedo model of the planetary

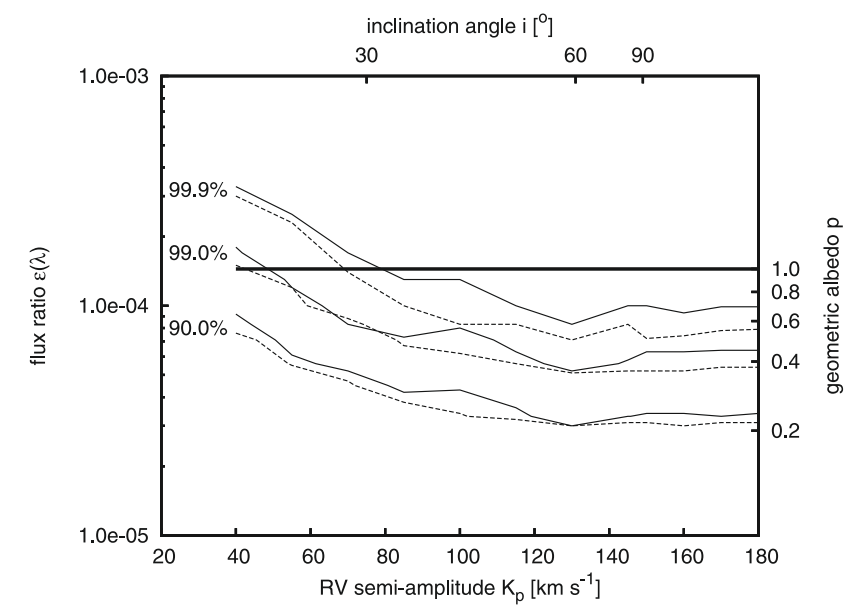

Fig. 3. Contour map showing confidence levels for the upper limits to the planet-to-star flux ratio $\epsilon(\lambda)$ as a function of to the RV semiamplitude $K_{\mathrm{p}}$ of the planet (lower $x$-axis), or orbital inclination $i$ (upper $x$-axis). From top to bottom, these levels are: $99.9,99.0$ and $90.0 \%$ confidence. For the planetary model, we assumed a Venus-like phase function as well as a Class IV albedo function (solid lines) and a grey albedo (dashed lines). The corresponding geometric albedo $p$ is shown under the assumption that $R_{\mathrm{p}}=1.2 R_{\mathrm{Jup}}$ (the right-hand $y$-axis). The region above the thick horizontal line is excluded because the geometric albedo of the planetary atmosphere would exceed 1 .

atmosphere (either grey albedo or Class IV model). For each pair of the parameters $K_{\mathrm{p}}$ and $\epsilon(\lambda)$, we determined the confidence level of the best-fit model to the artificial data via bootstrap randomisation.

This enables place upper limits to be established to the planet-to-star flux ratio $\epsilon(\lambda)$ for different confidence levels, as a function of the RV semi-amplitude $K_{\mathrm{p}}$. Figure 3 shows that the upper limits to the planet-to-star flux ratio decrease with increasing orbital inclination, which is a direct consequence of the illumination geometry. As can be seen from Fig. 3 (dashed lines), we have the highest sensitivity for detection of the planetary signal at high orbital inclinations with the formal numerical optimum found at an RV semi-amplitude $K_{\mathrm{p}}=129 \mathrm{~km} \mathrm{~s}^{-1}$ corresponding to $i=60^{\circ}$. At this inclination, the $99.9 \%$ confidence upper limit to the planet-to-star flux ratio for the grey albedo model is $\epsilon=6.7 \times 10^{-5}$, while it is $\epsilon=8.3 \times 10^{-5}$ for the Class IV model. In Fig. 3, we clearly see that the upper limits established by adopting the grey albedo model are deeper than the ones found with the Class IV model. A plausible explanation for this seems to be that in the grey albedo model, all the absorption lines have the same weight. On the other hand, in the Class IV model, the absorption lines in the red part of the observed spectrum have a lower weight than those in the blue part. Hence, fewer lines are effectively used for the analysis.

Assuming a planetary radius $R_{\mathrm{p}}=1.2 R_{\text {Jup }}$, we find that the $99.9 \%$ upper limit to the geometric albedo is $p<0.46$ for the grey albedo model, and $p<0.57$ for the Class IV model, at a wavelength of $\lambda=445 \mathrm{~nm}$, which corresponds to the center of gravity of the albedo function. For comparison, at this wavelength Jupiter's geometric albedo is $p=0.43$ (Karkoschka 1994). We note that the planetary radius of $R_{\mathrm{p}}=1.2 R_{\text {Jup }}$ is the average value of the five transiting hot Jupiters listed in Sect. 2.3. 
Table 3. Estimates for the observing times required for UVES to collect high-S/N data sufficient to detect, with $99.9 \%$ confidence, the reflected light from HD 75289Ab with a specific planet-to-star flux ratio $\epsilon$. Here we assume a Class V model for highly reflective case and the isolated Class IV albedo shape for the fainter case (cf. Fig. 1). The corresponding values of the albedo values $p$ are based on the assumption that the planetary radius is $R_{\mathrm{p}}=1.2 R_{\mathrm{Jup}}$.

\begin{tabular}{ccccl}
\hline \hline$\epsilon$ & $p$ & $N$ (spectra) & Time $(\mathrm{h})$ & Albedo model \\
\hline $5.7 \times 10^{-5}$ & 0.4 & 324 & 19.5 & Class V \\
$2.9 \times 10^{-5}$ & 0.2 & 1460 & 87.6 & isolated Class IV \\
\hline
\end{tabular}

In the case that the planetary radius of HD 75289Ab is similar to that of the transiting hot Jupiter HAT-P-1 b $\left(R_{\mathrm{p}}=1.36 R_{\text {Jup }}\right.$, Bakos et al. 2007), we find the geoemetric albedo to be $p<0.36$ and $p<0.45$, respectively for the grey albedo model and the Class IV model. This would enable us to rule out the Class V albedo model.

\section{Conclusions and outlook}

We reanalyzed 684 high-precision spectra of the star HD 75289A, obtained during four nights by another research group in 2003, in an attempt to detect starlight reflected from the known hot Jupiter.

(i) We found that the upper limits to the planet-to-star ratios established by Leigh et al. (2003a) were based on erroneous orbital phase information, and therefore needed to be corrected.

(ii) Our reanalysis using the correct orbital phases produced a non-detection of the reflected light of the planet. We therefore placed upper limits on the planet-to-star flux ratios for different possible orbital inclinations and confidence levels. These upper limits, however, were $\approx 60 \%$ higher than the ones placed by Leigh since the planet was not observed at its best visibility.

(iii) We furthermore determined values of the geometric albedo that was 2.9 times higher than the values established by Leigh et al. (2003a), since, due to limited information on planetary transits available in 2003, these authors assumed a planetary radius that was larger by $33 \%$ than our value.

(iv) The previous upper limit to the geometrical albedo of 0.12 established by Leigh et al. (2003) was by far the deepest one determined for hot Jupiters up to now. These authors suggested that a Class $\mathrm{V}$ atmospheric model can be ruled out for HD 75289Ab. For the value of the planetary radius adopted by us, $R_{\mathrm{p}}=1.2 R_{\mathrm{Jup}}$, we find that the amount of data was insufficient to rule out specific atmospheric models. However, if the radius of HD 75289Ab is similar to that of the transiting planet HAT-P-1 b $\left(R_{\mathrm{p}}=1.36 R_{\mathrm{Jup}}\right.$, Bakos et al. 2007), we can rule out the Class V albedo model.
To detect reflected starlight from the hot Jupiter HD 75289Ab, more high-precision data would be needed. Table 3 lists estimates of observing times required to detect, with $99.9 \%$ confidence, the reflected light of a planet with a Class V model and an isolated Class IV model, respectively. These calculations are based on the following assumptions: the fictitious observations are scheduled optimally with respect to phase ( $\phi=0.30$ to 0.45 and 0.55 to 0.70$)$. These observations are carried out by using the blue arm of UVES at the VLT/UT2 in Chile. The wavelength range is $\lambda=400.3$ to $527.0 \mathrm{~nm}$, and the exposure time is chosen in such a way that a maximum count rate of $40000 \mathrm{ADU} / \mathrm{px}$ is achieved (cf. Sect. 3). Table 3 demonstrates that we would require about $20 \mathrm{~h}$ of observations to measure the reflected light of HD 75289Ab in the case that it had a Class $\mathrm{V}$ albedo function and a planetary radius of $R_{\mathrm{p}}=1.2 R_{\mathrm{Jup}}$. However, if HD 75289Ab had an albedo function similar to that of the isolated Class IV model, it would already take more than $87 \mathrm{~h}$ of observing time to detect the reflected light.

Because of the large amount of data required, we conclude that the star HD 75289A is too faint for the search for reflected starlight. Instead, the search for thermal emission of $\mathrm{HD} 75289 \mathrm{Ab}$ in the near-infrared appears to be promising, since the planet-to-star flux ratio increases dramatically in the infrared (Barnes et al. 2007).

Acknowledgements. We are grateful to Tsevi Mazeh for valuable discussions. Furthermore, we want to thank the anonymous referee for valuable comments. Based on observations made with ESO Telescopes at the Paranal Observatory under programme ID 70.C-0061(A).

\section{References}

Bakos, G., Noyes, R., Kovácz, G., Latham, D., et al. 2007, ApJL, 656, 552 Barnes, J. R., Leigh, C. J., Jones, H. R. A., et al. 2007, MNRAS, 379, 1097 Burrows, A., Hubeny, I., Budaj, J., \& Hubbard, W. B. 2007, ApJ, 661, 502 Butler, R. P., Wright, J. T., Marcy, G. W., et al. 2006, ApJ, 646, 505 Charbonneau, D., Noyes, R. W., Korzennik, S. G., et al. 1999, ApJ, 522, L145 Gratton, R. G., Focardi, P., \& Bandiera, R. 1989, MNRAS, 237, 1085

Hilton, J. L. 1992, Explanatory Supplement to the Astronomical Almanac (Mill Valley CA: Universiy Science Books), 383

Karkoschka, E. 1994, Icarus, 111, 174

Kürster, M., Schmitt, J. H. M. M., Cutispoto, G., \& Dennerl, K. 1997, A\&A, 320,831

Leigh, C., Collier Cameron, A., Horne, K., \& Penny, A. 2003a, MNRAS, 344, 1271

Leigh, C., Collier Cameron, A., \& Guillot, T. 2003b, MNRAS, 346, 890

Mugrauer, M., Neuhäuser, R., Mazeh, T., \& Alves, J. 2004, A\&A, 425, 249

Press, W. H., Teukolsky, S. A., Vetterling, W. T., \& Flannery, B. P. 1992, Numerical recipes in C. The art of scientific computing (Cambridge University Press)

Seager, S., \& Sasselov, D. D. 1998, ApJ, 502, 157

Sudarsky, D., Burrows, A., \& Pinto, P. 2000, ApJ, 538, 885

Sudarsky, D., Burrows, A., \& Hubeny, I. 2003, ApJ, 588, 1121

Udry, S., Mayor, M., Naef, D., Pepe, F., \& Queloz, D. 2000, A\&A, 356, 590

Valenti, J. A., \& Fischer, D. A. 2005, ApJS, 159, 141 